\title{
On the Local and Stratified Likelihood Approaches in Single-Index Hazards Model
}

\author{
Kai Ding • Michael R. Kosorok • Donglin Zeng
}

Received: 5 May 2013 / Accepted: 17 June 2013 / Published online: 20 July 2013

(C) School of Mathematical Sciences, University of Science and Technology of China and Springer-Verlag Berlin Heidelberg 2013

\begin{abstract}
We propose the single-index hazards model for censored survival data. As an extension of the Cox model and many transformation models, this model allows nonparametric modeling of covariate effects in a parsimonious way via a single index. In addition, the relative importance of covariates can be assessed via this model. We consider two commonly used profile likelihood methods for parameter estimation: the local profile likelihood method and the stratified profile likelihood method. It is shown that both methods may give consistent estimators under certain restrictive conditions, but in general they can yield biased estimation. Simulation studies are also conducted to demonstrate these bias phenomena. The existence and nature of the failures of these two commonly used approaches is somewhat surprising.
\end{abstract}

Mathematics Subject Classification (2010) 62N01

Keywords Bias analysis · Cox model · Local likelihood · Profile likelihood · Single-index $\cdot$ Stratification

\section{Introduction}

The Cox [6] proportional hazards model is widely used in survival analysis. In this model, the conditional hazard rate of failure time given covariates, denoted by $Z$, is modeled as $h(t \mid Z)=\lambda(t) e^{\gamma^{T} Z}$, where $\lambda(\cdot)$ is a completely unknown baseline hazard

K. Ding · M.R. Kosorok · D. Zeng $(\varangle)$

Department of Biostatistics, University of North Carolina, Chapel Hill, NC 27599-7420, USA

e-mail: dzeng@bios.unc.edu

K. Ding

e-mail: kding@bios.unc.edu

M.R. Kosorok

e-mail: kosorok@unc.edu 
function. The regression parameter, $\gamma$, can be nicely interpreted as the log-hazard ratios of the covariates $Z$. Cox [7] also proposed the partial likelihood to estimate the regression parameters. The large sample properties of the partial likelihood estimators were later proved in Andersen and Gill [2]. See also Fleming and Harrington [11] and Andersen et al. [1] for the literature concerning this model.

However, in many real data sets, covariates may exhibit much more complicated effects than log-linear effects; thus, the proportional hazards assumption may be violated. For this reason, many authors have considered some alternatives or extensions of the Cox model including the accelerated failure time model [8], the proportional odds model [3, 17], the proportional hazards frailty regression models [14] and transformation models [19].

Other extensions of the Cox model include the fully nonparametric model of the form $h(t \mid Z)=\lambda(t, Z)$ studied by Nielsen and Linton [16], where the function $\lambda(\cdot, \cdot)$ is completely unknown. A kernel estimator was proposed by maximizing a local likelihood function. The uniform convergence and the asymptotic normality of the proposed estimator were established. Later, Fan et al. [10] studied a multiplicative nonparametric model of the form $h(t \mid Z)=\lambda(t) e^{\phi(Z)}$, where the logarithm of the hazard rate function is assumed to be the sum of an unknown function of covariates and an unknown function of the survival time. The estimator of $\phi(\cdot)$ was constructed by maximizing a local likelihood function as well. Although these two models can flexibly model covariate effects, the limitation is that the nonparametric estimation is only practically feasible when the dimension of $Z$ is low.

One of the most convenient models commonly used in biometrics and econometrics for dimension reduction is the single-index model discussed by Härdle et al. [12]. The model takes the form $Y=\phi\left(\gamma^{T} Z\right)+\epsilon$, where $\phi(\cdot)$ is an unknown function, $\gamma$ is the orientation vector and $\epsilon$ is the random error. The linear combination $\gamma^{T} Z$ is called a single index. A kernel smoothing method is used to estimate $\phi(\cdot)$ and the estimator of $\gamma$ minimizes a modified mean square error function. Instead of considering all nonparametric covariate effects, Carroll et al. [4] proposed the generalized partially linear single-index model where a local quasi-likelihood was used to estimate the unknown function of the single index.

In this paper, we consider a modification of Nielsen and Linton [16] by assuming a nonparametric baseline hazard function that depends on $Z$ through a single in$\operatorname{dex} \gamma^{T} Z$. Specifically, we consider a model of the form $h(t \mid Z)=\lambda\left(t, \gamma^{T} Z\right)$, where $\lambda(\cdot, \cdot)$ is an unknown function. Note that this model includes the Cox model and all the transformation models mentioned before as special cases. In addition, the model has several nice features. First, covariates are allowed to have nonparametric effects on the hazard function. This is particularly useful if covariates $Z$ do not satisfy the proportional hazards assumption so that the Cox model may not be appropriate. Second, the relative importance of the components of $Z$ can be fully characterized by the orientation vector $\gamma$ since the derivative of $h(t \mid Z)$ with respect to $Z_{i}$, the $i$ th component of the covariate vector $Z$, is proportional to $\gamma_{i}$, thus $\gamma_{i}$ characterizes how fast $h(t \mid Z)$ changes with $Z_{i}$. Third, our model is more parsimonious than the model in Nielsen and Linton [16] since the multi-dimensional vector $Z$ has been replaced by a one-dimensional single index $\gamma^{T} Z$.

The local likelihood approach is commonly used for the single-index model. Thus, in this paper, we adapt this approach for parameter estimation in our single-index 
hazards model. Surprisingly, we find, both theoretically and numerically, that this commonly used approach in general yields inconsistent estimators and it works only under very specific conditions. Also, we consider another popular approach in epidemiologic studies, named the stratified likelihood approach, and we obtain a similar surprising conclusion.

This paper focuses on the bias analysis on the local likelihood approach and the stratified likelihood approach and it is organized as follows. In Sect. 2, we describe the single-index hazards model and the data structure. In Sect. 3, we describe how to adapt the commonly used local profile likelihood method for parameter estimation. We then study the asymptotic bias of this approach in Sect. 4 and identify conditions under which this approach may work. In Sect. 5, we introduce the stratified likelihood approach and carry out a similar bias analysis. In Sect. 6, we demonstrate our findings via a series of simulation studies and finally we conclude with a discussion in Sect. 7.

\section{Model and Data Structure}

We assume the following single-index hazards model:

$$
h(t \mid Z)=\lambda\left(t, \gamma^{T} Z\right),
$$

where $\gamma \in \mathbb{R}^{q}$ and $\lambda(\cdot, \cdot)$ is an unknown bivariate function. To ensure identifiability, it is necessary to impose the restriction that $\|\gamma\|=1$ with one non-zero component positive, that is, the $\gamma$ vector is restricted to the half unit sphere. This assumption is practically reasonable when at least one covariate has a non-zero effect. Furthermore, if one knows one component of $\gamma$, say the first component denoted by $\gamma_{1}$, to be nonzero, then an equivalent model to (1) is to redefine $\gamma$ so that $\gamma_{1}=1$ and the new $\lambda$-function as $\lambda\left(t, \gamma_{1} w\right)$. Under the equivalent model, only $\lambda(\cdot, \cdot)$ and the components of $\gamma$ except $\gamma_{1}$ are the unknown parameters and there are not any constraints for $\gamma$.

Suppose we observe a random sample of size $n,\left(Y_{i}=T_{i} \wedge C_{i}, \Delta_{i}, Z_{i}\right)$, $i=1, \ldots, n$, where $T$ is the survival time, $C$ is the censoring time, $a \wedge b=\min (a, b)$, $\Delta=I(T \leq C)$ is the censoring indicator and $Z$ is the covariate vector. The subscript $i$ is used to denote the $i$ th subject. The log-likelihood function is

$$
\frac{1}{n} \sum_{i=1}^{n}\left[\Delta_{i} \log \lambda\left(Y_{i}, \gamma^{T} Z_{i}\right)-\Lambda\left(Y_{i}, \gamma^{T} Z_{i}\right)\right] .
$$

This function has a maximum value of infinity and thus cannot be used directly for parameter estimation. In nonparametric maximum likelihood estimation (NPMLE), we maximize

$$
\frac{1}{n} \sum_{i=1}^{n}\left[\Delta_{i} \log \Lambda\left\{Y_{i}, \gamma^{T} Z_{i}\right\}-\sum_{Y_{j} \leq Y_{i}} \Lambda\left\{Y_{j}, \gamma^{T} Z_{i}\right\}\right]
$$

Here, $\Lambda\left\{Y_{i}, \gamma^{T} Z_{i}\right\}$ is the jump size of $\Lambda\left(Y_{i}, \gamma^{T} Z_{i}\right)$ at $Y_{i}$. However, the profile likelihood function based on (3), obtained by profiling out $\Lambda\{\cdot, \cdot\}$, is a constant and is thus not a valid objective function. In the next section, we consider a commonly used estimation approach for model (1), the local profile likelihood approach. 


\section{Local Likelihood Approach}

Local likelihood has been frequently used to estimate the unknown function in a semiparametric model. In this approach, a local likelihood is constructed to estimate the nonparametric function and then the estimated function is plugged into the likelihood to obtain the profile likelihood function. This conventional profile-kernel method was adopted by Fan et al. [10]. Carroll et al. [4] used the same method except that a quasilikelihood played the role of the regular likelihood function. Specifically for our likelihood (3) and fixed $\gamma$, we would estimate $\Lambda\{\cdot, \cdot\}$ by maximizing the following local likelihood:

$$
\frac{1}{n} \sum_{i=1}^{n}\left[\Delta_{i} \log \Lambda\left\{Y_{i}, w\right\}-\sum_{Y_{j} \leq Y_{i}} \Lambda\left\{Y_{j}, w\right\}\right] K_{a_{n}}\left(\gamma^{T} Z_{i}-w\right)
$$

where $K_{a_{n}}(t)=K\left(t / a_{n}\right) / a_{n}, K$ is a mean zero symmetric density function and $\Lambda\left\{Y_{i}, w\right\}$ is the jump size of $\Lambda\left(Y_{i}, w\right)$ at $Y_{i}$ for each $w$. This is the local constant fit weighted by the function $K_{a_{n}}(\cdot)$. The maximizer can be found as

$$
\hat{\Lambda}\left\{Y_{i}, w\right\}=\frac{\Delta_{i} K_{a_{n}}\left(\gamma^{T} Z_{i}-w\right)}{\sum_{Y_{j} \geq Y_{i}} K_{a_{n}}\left(\gamma^{T} Z_{j}-w\right)} .
$$

After plugging (4) into (3), we find, up to a constant, that the local profile likelihood is

$$
\begin{aligned}
- & \frac{1}{n} \sum_{i=1}^{n} \Delta_{i} \log \left(\frac{1}{n a_{n}} \sum_{Y_{j} \geq Y_{i}} K\left(\frac{\gamma^{T}\left(Z_{j}-Z_{i}\right)}{a_{n}}\right)\right) \\
& -\frac{1}{n} \sum_{i=1}^{n} \frac{1}{n a_{n}} \sum_{Y_{j} \leq Y_{i}} \frac{\Delta_{j} K\left(\frac{\gamma^{T}\left(Z_{j}-Z_{i}\right)}{a_{n}}\right)}{\frac{1}{n a_{n}} \sum_{Y_{k} \geq Y_{j}} K\left(\frac{\gamma^{T}\left(Z_{k}-Z_{i}\right)}{a_{n}}\right)} \\
= & (A)-(B) .
\end{aligned}
$$

We will show that term $(B)$ equals a constant asymptotically in the next section. As a result, the estimator of $\gamma$ is the maximizer of the local profile likelihood function $p l_{n}^{\mathrm{loc}}(\gamma)$, which only includes the first term $(A)$. That is,

$$
p l_{n}^{\mathrm{loc}}(\gamma)=-\frac{1}{n} \sum_{i=1}^{n} \Delta_{i} \log \left(\frac{1}{n a_{n}} \sum_{Y_{j} \geq Y_{i}} K\left(\frac{\gamma^{T}\left(Z_{j}-Z_{i}\right)}{a_{n}}\right)\right) .
$$

Note this function is smooth in $\gamma$. Thus numerically it can be easily maximized. For example, the quasi-Newton search algorithm under constraint $\|\gamma\|=1$ can be used. Alternatively, if we know the first component of $\gamma, \gamma_{1}$, to be non-zero, then the maximization can be carried out by setting $\gamma_{1}=1$ and unconstrained optimization is used to maximize the above function over the rest components of $\gamma$. The obtained estimator is then normalized to ensure $\|\gamma\|=1$. Finally, different initial values or grid search will be used in the optimization to ensure that a global maximizer is found. 


\section{Bias Analysis in the Local Likelihood Approach}

In this section, we aim to rigorously study the estimation bias based on the local profile likelihood $p l_{n}^{\text {loc }}(\gamma)$. We impose the following regularity conditions:

- (C1) $\gamma_{0} \in \Gamma$, where $\Gamma \in \mathbb{R}^{q}$ is compact.

- (C2) The random covariate vector $Z$ has a continuous density on its support.

- (C3) The non-uniform kernel function $K(\cdot)$ has zero mean with finite second moment. Moreover, $\sup _{x}\left|K^{\prime}(x)\right|$ is finite, where $K^{\prime}(x)$ denotes the derivative function of $K(x)$.

- (C4) The bandwidth $a_{n}=n^{\nu_{1}}$ with $v_{1} \in(-1 / 2,0)$.

Remark 1 Many kernel functions satisfy condition (C3), for example, the standard Gaussian kernel $K(u)=1 / \sqrt{2 \pi} \exp \left(-u^{2} / 2\right)$ and the Epanechnikov kernel $K(u)=$ $3 / 4\left(1-u^{2}\right) I(|u| \leq 1)$.

Our first theorem establishes that term $(B)$ equals a constant asymptotically, and thus is negligible. Our second theorem gives the asymptotic limit of $p l_{n}^{\text {loc }}(\gamma)$.

Theorem 1 If conditions (C1)-(C4) hold, then $\sup _{\gamma}\left|(B)-1 / n \sum_{j=1}^{n} \Delta_{j}\right| \rightarrow{ }_{\text {a.s. }} 0$.

Theorem 2 If conditions $(\mathrm{C} 1)-(\mathrm{C} 4)$ hold, then $\sup _{\gamma}\left|p l_{n}^{\mathrm{loc}}(\gamma)-p l^{\mathrm{loc}}(\gamma)\right| \rightarrow{ }_{\text {a.s. }} 0$, where

$$
p l^{\mathrm{loc}}(\gamma)=-E\left[\Delta \log \left(\left.P\left(Y \geq y \mid \gamma^{T} Z\right)\right|_{y=Y} f_{\gamma^{T} Z}\left(\gamma^{T} Z\right)\right)\right] .
$$

Here $f_{\gamma^{T} Z}(\cdot)$ is the density function of $\gamma^{T} Z$.

Thus the local profile likelihood estimator should converge to the maximizer of $p l^{\text {loc }}(\gamma)$ almost surely by Theorem 2.12 of Kosorok [13]. Suppose the latter is the true parameter $\gamma_{0}$, then the derivative of $p l^{\text {loc }}(\gamma)$ with respect to $\gamma$ should be proportional to $\gamma_{0}$ if evaluated at $\gamma_{0}$. This proportionality to $\gamma_{0}$ is due to the restriction $\|\gamma\|=1$. However, we show in Theorem 3 that this may not be true under the following two regularity conditions:

- (C5) Given covariates $Z, T$ and $C$ are independent.

- (C6) $P(T>\tau)<1$, where $\tau$ denotes the end of the study.

Remark 2 Condition (C6) implies a positive probability of non-censoring so that $p l_{n}^{\text {loc }}(\gamma)$ is not a constant with respect to $\gamma$.

Theorem 3 Assume conditions (C5) and (C6) hold and suppose $C$ is independent of $Z$ and $Z \sim N(\mu, \Sigma)$ with $\Sigma$ positive definite, then $\left.\frac{\partial}{\partial \gamma}\right|_{\gamma=\gamma_{0}} p l^{\mathrm{loc}}(\gamma) \propto \gamma_{0}$ if and only if $\Sigma \gamma_{0}=c \gamma_{0}$ for some constant $c$.

Remark 3 This theorem suggests that even in the special case where the covariate vector follows a normal distribution and $C$ is independent of $Z$, the local profile likelihood approach may give consistent estimation only under the restrictive condition 
$\Sigma \gamma_{0}=c \gamma_{0}$. Thus, in the most general set-up, $p l^{\mathrm{loc}}(\gamma)$ may not be maximized at $\gamma_{0}$, and thus the procedure may be inconsistent.

\section{Stratified Likelihood Approach}

For a fixed $\gamma$, model (1) can be viewed as a stratified hazards model with strata defined by values of $\gamma^{T} Z$. The model is in spirit similar to the stratified Cox model, which is commonly used in epidemiologic studies, such as in Motzer et al. [15] and Chow et al. [5]. This similarity also allows us to consider the following stratified profile likelihood approach.

For any fixed $\gamma$, we stratify the range $R$ of $\gamma^{T} Z_{i}, i=1, \ldots, n$ based on the sample quantiles. Specifically, let $\min _{1 \leq i \leq n} \gamma^{T} Z_{i}=t_{0}<\cdots<t_{J_{n}}=\max _{1 \leq i \leq n} \gamma^{T} Z_{i}$ be a partition of $R$ into $J_{n}$ subintervals $\left[t_{k-1}, t_{k}\right), k=1, \ldots, J_{n}$ such that $t_{k}=\mathbb{F}_{n}^{-1}\left(k / J_{n}\right)$, where $\mathbb{F}_{n}(\cdot)$ is the empirical distribution of $\gamma^{T} Z_{i}, i=1, \ldots, n$. Note that $t_{i}$ is a random variable depending on $\gamma$. Also, it is clear by the above construction that $I\left(\gamma^{T} Z_{i} \in\left[t_{k-1}, t_{k}\right)\right)=I\left(\mathbb{F}_{n}\left(\gamma^{T} Z_{i}\right) \in\left[(k-1) / J_{n}, k / J_{n}\right)\right)$. Let $S_{k} \equiv\left[(k-1) / J_{n}, k / J_{n}\right)$. We assume for any $(t, u)$,

$$
\lambda(t, u)=\sum_{k=1}^{J_{n}} I\left(u \in\left[t_{k-1}, t_{k}\right)\right) \lambda_{k}(t)=\sum_{k=1}^{J_{n}} I\left(\mathbb{F}_{n}(u) \in S_{k}\right) \lambda_{k}(t),
$$

thus we assume the baseline hazard function takes different forms on different strata defined by the sample quantiles of $\gamma^{T} Z$. Plug this into (2) and then in the setting of the NPMLE, we maximize

$$
\begin{gathered}
\frac{1}{n} \sum_{i=1}^{n}\left[\Delta_{i} \sum_{k=1}^{J_{n}} \log \Lambda_{k}\left\{Y_{i}\right\} I\left(\mathbb{F}_{n}\left(\gamma^{T} Z_{i}\right) \in S_{k}\right)\right. \\
\left.\quad-\sum_{k=1}^{J_{n}} I\left(\mathbb{F}_{n}\left(\gamma^{T} Z_{i}\right) \in S_{k}\right) \sum_{Y_{j} \leq Y_{i}} \Lambda_{k}\left\{Y_{j}\right\}\right] .
\end{gathered}
$$

The maximizer for $\Lambda_{k}\left\{Y_{i}\right\}$ is

$$
\hat{\Lambda}_{k}\left\{Y_{i}\right\}=\frac{\Delta_{i} I\left(\mathbb{F}_{n}\left(\gamma^{T} Z_{i}\right) \in S_{k}\right)}{\sum_{Y_{j} \geq Y_{i}} I\left(\mathbb{F}_{n}\left(\gamma^{T} Z_{j}\right) \in S_{k}\right)} .
$$

After plugging (6) into (5), we find, up to a constant, that the stratified profile likelihood function is

$$
p l_{n}^{s}(\gamma)=-\frac{1}{n} \sum_{i=1}^{n} \Delta_{i} \sum_{k=1}^{J_{n}}\left[I\left(\mathbb{F}_{n}\left(\gamma^{T} Z_{i}\right) \in S_{k}\right) \log \left(\frac{J_{n}}{n} \sum_{Y_{j} \geq Y_{i}} I\left(\mathbb{F}_{n}\left(\gamma^{T} Z_{j}\right) \in S_{k}\right)\right)\right] .
$$

Note that $p l_{n}^{s}(\gamma)$ is not smooth in $\gamma$ so that it is numerically difficult to find its maximizer. In the next section, we use the grid search to find the maximum point. However, the grid search becomes infeasible when the dimension of $Z$ increases. 
Let $\hat{\gamma}_{n}$ denote the maximizer of $p l_{n}^{s}(\gamma)$. We now study the asymptotic property of $\hat{\gamma}_{n}$ by first obtaining the asymptotic limit $p l^{s}(\gamma)$ of $p l_{n}^{s}(\gamma)$ given in Theorem 4 . The following regularity conditions are further imposed:

- (C7) $J_{n} / \sqrt{n} \rightarrow 0, J_{n} \rightarrow \infty$.

- (C8) $\lambda_{0}(t, u)$ has non-zero partial derivative with respect to $u$ for any $t \in[0, \tau]$; moreover, $\int_{0}^{\tau} \lambda_{0}(t, u) d t<\infty$, for any $u \in \mathbb{R}$.

Theorem 4 If conditions (C1) and (C7) hold, then $\sup _{\gamma}\left|p l_{n}^{s}(\gamma)-p l^{s}(\gamma)\right| \rightarrow_{P} 0$, where

$$
\begin{aligned}
p l^{s}(\gamma) & =-E\left[\left.\Delta \log P\left(Y \geq y \mid F_{\gamma^{T} Z}\left(\gamma^{T} Z\right)\right)\right|_{y=Y}\right] \\
& =-E\left[\left.\Delta \log P\left(Y \geq y \mid \gamma^{T} Z\right)\right|_{y=Y}\right] .
\end{aligned}
$$

Here $F_{\gamma^{T} Z}(\cdot)$ is the distribution function of $\gamma^{T} Z$.

Note that the difference between $p l^{\text {loc }}(\gamma)$ and $p l^{s}(\gamma)$ is that the latter does not involve the density $f_{\gamma^{T} Z}\left(\gamma^{T} Z\right)$ in the log function. The reason is that the stratified method is based on $F_{\gamma^{T} Z}\left(\gamma^{T} Z\right)$ which follows the uniform distribution on [0,1].

From Theorem 4, we conclude similarly that $\hat{\gamma}_{n}$ converges to the maximizer of $p l^{s}(\gamma)$ in probability. Next, we show in Theorem 5 that $\hat{\gamma}_{n}$ is consistent for $\gamma_{0}$.

Theorem 5 Assume conditions (C1) and (C5)-(C8) hold and suppose $C$ is independent of $Z$, then $\hat{\gamma}_{n} \rightarrow P \gamma_{0}$.

Remark $4 \partial / \partial u \lambda_{0}(t, u)=0$ implies $\lambda_{0}(t, u)$ is constant in $u$ and thus $Z$ has no effect on the hazard function. Therefore, assuming the first part of condition (C8) is not unreasonable. The second part of condition (C8) ensures a positive probability of censoring. Also, we have again assumed the independence between $C$ and $Z$. Without this assumption, we conjecture that the stratified likelihood approach would lead to biased estimation. In fact, the simulation studies in the next section suggest that this approach can fail if $C$ and $Z$ are dependent.

\section{Simulation Studies}

We conduct numerical studies to demonstrate the estimation bias associated with two aforementioned profile likelihood approaches. In this section, we assume that the covariate vector $Z=\left(Z_{1}, Z_{2}\right)$ is two dimensional and is generated from a bivariate normal distribution with zero means and unit variances. The true parameter for $\gamma$ is $\gamma_{0}=(-1 / 2, \sqrt{3} / 2)^{T}$. The following four simulation settings are considered: (i) The censoring time $C$ is independent of $Z, \lambda_{0}(t, u)=0.5 e^{u} t$, $Z$ has no correlation; (ii) $C$ is independent of $Z, \lambda_{0}(t, u)=0.5 e^{u} t$ and the covariance between $Z_{1}$ and $Z_{2}$ is 0.5 ; (iii) $C$ is independent of $Z, \lambda_{0}(t, u)=$ $0.25\left(t+u^{2}\right)$ and we use the same covariance matrix as in setting (ii); (iv) $C$ and $Z$ are dependent, $\lambda_{0}(t, u)=0.25\left(t+u^{2}\right)$ and we use the same covariance matrix as 
Table 1 Summary of simulation results, each entry is based on 500 replicates

\begin{tabular}{|c|c|c|c|c|c|c|c|}
\hline & \multirow[t]{2}{*}{$n$} & \multicolumn{2}{|c|}{ Local likelihood } & \multicolumn{2}{|c|}{ Stratified likelihood } & \multicolumn{2}{|c|}{ Cox model } \\
\hline & & Bias & SE & Bias & SE & Bias & SE \\
\hline \multicolumn{8}{|c|}{ Simulation (i): $C$ independent of $Z, \lambda_{0}(t, u)=0.5 e^{u} t$, correlation 0 in $Z$} \\
\hline \multirow[t]{3}{*}{$\gamma_{1}$} & 2000 & 0.153 & 0.421 & 0.003 & 0.076 & -0.001 & 0.026 \\
\hline & 5000 & 0.018 & 0.183 & -0.000 & 0.059 & 0.000 & 0.017 \\
\hline & 10,000 & 0.013 & 0.113 & -0.001 & 0.039 & 0.000 & 0.012 \\
\hline \multicolumn{8}{|c|}{ Simulation (ii): $C$ independent of $Z, \lambda_{0}(t, u)=0.5 e^{u} t$, correlation 0.5 in $Z$} \\
\hline \multirow[t]{3}{*}{$\gamma_{1}$} & 2000 & 1.198 & 0.035 & 0.001 & 0.066 & -0.001 & 0.030 \\
\hline & 5000 & 1.195 & 0.023 & 0.004 & 0.049 & 0.000 & 0.019 \\
\hline & 10,000 & 1.195 & 0.017 & 0.002 & 0.034 & 0.001 & 0.014 \\
\hline \multicolumn{8}{|c|}{ Simulation (iii): $C$ independent of $Z, \lambda_{0}(t, u)=0.25\left(t+u^{2}\right)$, correlation 0.5 in $Z$} \\
\hline \multirow[t]{3}{*}{$\gamma_{1}$} & 2000 & 1.188 & 0.037 & 0.015 & 0.121 & 0.500 & 0.032 \\
\hline & 5000 & 1.189 & 0.022 & 0.008 & 0.078 & 0.501 & 0.020 \\
\hline & 10,000 & 1.185 & 0.017 & 0.003 & 0.057 & 0.500 & 0.014 \\
\hline \multicolumn{8}{|c|}{ Simulation (iv): $C$ depends on $Z, \lambda_{0}(t, u)=0.25\left(t+u^{2}\right)$, correlation 0.5 in $Z$} \\
\hline \multirow[t]{3}{*}{$\gamma_{1}$} & 2000 & 1.338 & 0.027 & -0.312 & 0.030 & 0.446 & 0.033 \\
\hline & 5000 & 1.340 & 0.016 & -0.310 & 0.018 & 0.448 & 0.022 \\
\hline & 10,000 & 1.342 & 0.011 & -0.310 & 0.014 & 0.447 & 0.015 \\
\hline
\end{tabular}

in setting (ii). In settings (i)-(iii), $C$ is generated from the uniform $[0, \tau]$ distribution with $\tau=10$ and $C=4 e^{Z_{2}} \wedge \tau$ in setting (iv). Note that in setting (i) and (ii), the proportional hazards assumption is satisfied, but this assumption is violated in settings (iii) and (iv). The censoring rate ranges approximately from $20 \%$ to $28 \%$.

For the local likelihood approach, we choose the kernel function to be the standard normal density and the parameter is estimated by using the quasi-Newton search algorithm in the $\mathrm{R}$ software package. The initial value is set to zero. The bandwidth is chosen to be $c_{1} \times I Q R_{1} \times n^{-1 / 4}$, where the tuning parameter $c_{1}$ is chosen from $\{2,1,1 / 2\}$ and $I Q R_{1}$ is the inter-quartile range of $\|Z\|$ in each simulated data set. For each simulation setting, the tuning parameter $c_{1}$ which gives the smallest bias when $n=10,000$ is chosen and then the same parameter value is used for other sample sizes.

For the stratified approach, a grid search with step size 0.01 is used since the objective function is not continuous in $\gamma$. The number of strata is chosen from 4,8 or 12 for $\gamma^{T} Z$. We report the results from the number of strata yielding the smallest bias.

Table 1 summarizes the simulation results in setting (i)-(iv) with sample sizes 2000, 5000 and 10,000, where $\gamma_{1}$ is the first component of the $\gamma$ vector. As expected by Theorem 3, the local likelihood approach fails in settings (ii)-(iv) due to the correlation among the vector $Z$ and $\gamma_{0}$ not being an eigenvector of the covariance matrix of $Z$. Theorem 3 also suggests that the local likelihood approach may work in setting (i) because the identity covariance matrix is used. As expected by Theorem 5, the 

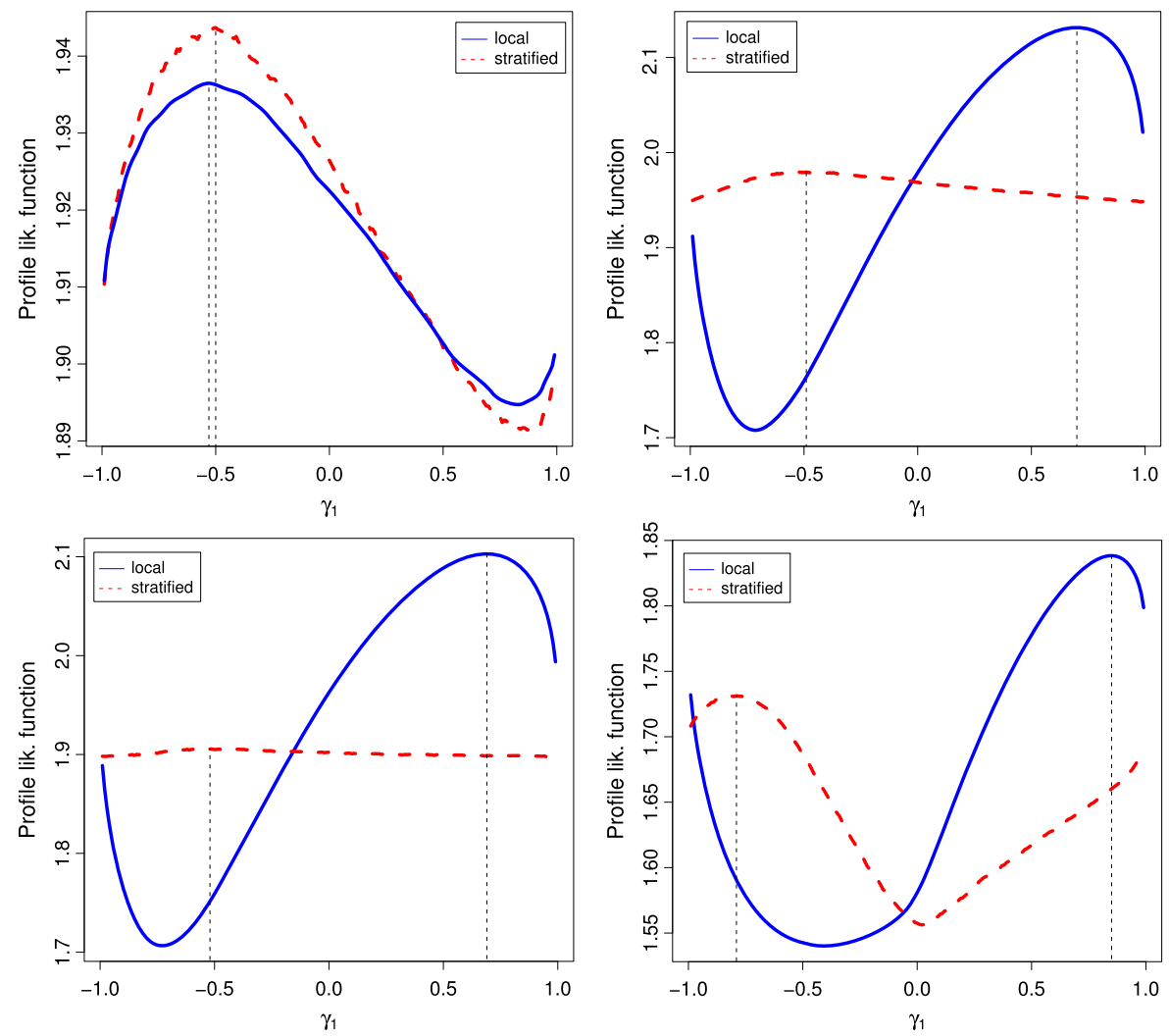

Fig. 1 Profile log-likelihood function of $\gamma_{1}$ for a simulated data set with $n=10,000$ : The upper two pertain to case (i) and (ii), respectively; The bottom two pertain to case (iii) and (iv), respectively. The bandwidth is $1 \times n^{-1 / 4}$. The number of strata is 12

stratified approach works in simulation settings (i)-(iii). However, it fails in setting (iv) probably due to the dependence between $C$ and $Z$. We have also reported the results from the Cox proportional hazards model. The Cox model produces consistent estimators in setting (i) and (ii) since the proportional hazards assumption is satisfied, but it gives biased estimation in setting (iii) and (iv) due to the violation of this assumption. Figure 1 shows the profile likelihood functions in each simulation setting based on one single simulated data set of size 10,000. Again, the local likelihood approach gives biased estimators except in setting (i). The stratified profile likelihood curves are maximized around the true value -0.5 of $\gamma_{1}$ in the first three simulation settings, suggesting that the stratified approach yields estimators with little bias in these settings, but it gives biased estimation in setting (iv) where $C$ depends on $Z$.

\section{Discussion}

We have considered two commonly used profile likelihood approaches for our singleindex hazards model. Even under the restrictive condition that the censoring time $C$ 
and the covariate vector $Z$ are independent, the local profile likelihood method gives inconsistent estimation in general. Therefore, this method should not be used for our model. In contrast, under this independent censoring assumption and some other regularity conditions, the stratified profile likelihood method always yields consistent estimation.

The main difference between the local likelihood and the stratified approach is the way of estimating $\lambda\left(t, \gamma^{T} Z\right)$ using observations in different ways. The local likelihood is constructed to estimate the unknown hazard function. The estimator given in (4) reveals that "local" data are given more weights than "remote" data. This unequally weighted structure causes bias, intuitively. In contrast, under the stratified likelihood approach, the estimator of the hazard function given in (6) has the property that data within the same stratum are equally weighted. We note that the stratification needs to be based on sample quantiles of the single index since an equally spaced stratification on the original scale of the single-index would lead to the same limiting profile likelihood function as under the local likelihood approach and thus the same estimation bias would occur.

In addition to the independent censoring assumption, another requirement in order for the stratified likelihood approach to be a consistent procedure is a positive probability of censoring in the data, as guaranteed by the second part of condition (C8). If there is no censoring present, it can be shown that $p l_{n}^{s}(\gamma)$ is free of $\gamma$ and converges to the constant 1 (the limit function $p l^{S}(\gamma) \equiv 1$ as well), and thus cannot be used for parameter estimation. That the presence of censoring is required to achieve consistency is quite surprising.

We note that the independent censoring assumption is crucial for the stratified likelihood approach to work. Without this assumption, we conjecture that the stratified likelihood method fails, as demonstrated numerically in Sect. 6. Therefore, one should not use the stratified likelihood approach either unless the independent censoring can be reasonably assumed. One possible way to relax this restrictive assumption is to modify the limit stratified profile likelihood function so that the modified function has a unique maximizer at the true parameter value without assuming the independence between $C$ and $Z$. We then can make a corresponding modification in the original stratified profile likelihood function and use it for estimation.

Besides the aforementioned methods, the spline method can also possibly be used for parameter estimation in our single-index hazards model. For example, Yu and Ruppert [18] considered the penalized spline method in a partially linear singleindex model and they showed that their method outperforms the local likelihood method adopted by Carroll et al. [4]. It would be worthwhile to examine a spline method for our model and to investigate whether or not the estimation bias issue still exists.

The existence and nature of the failures of the two commonly used estimation approaches considered in this paper are somewhat surprising and suggest that nonstandard approaches may be needed. In addition to the aforementioned spline approach, there are yet other approaches which may need to be considered in order to find an estimator that is consistent under realistically general conditions. 


\section{Appendix}

Proof of Theorem 1 We partition $\Gamma$ into small cubes such that any two points in the same cube have distance no large than $\delta_{n}$ to be determined later. The number of partitions, denoted by $m_{n}^{*}$, is of order $1 / \delta_{n}^{q}$. Choose one arbitrary point from each of these partitions and denote them as $\gamma^{(1)}, \ldots, \gamma^{\left(m_{n}^{*}\right)}$. For $\gamma_{1}$ and $\gamma_{2}$ in the same cube, any fixed $y, z$,

$$
\left|\frac{1}{n a_{n}} \sum_{Y_{j} \geq y} K\left(\frac{\gamma_{1}^{T}\left(Z_{j}-z\right)}{a_{n}}\right)-\frac{1}{n a_{n}} \sum_{Y_{j} \geq y} K\left(\frac{\gamma_{2}^{T}\left(Z_{j}-z\right)}{a_{n}}\right)\right| \leq \frac{c}{a_{n}^{2}}\left\|\gamma_{1}-\gamma_{2}\right\|,
$$

and

$$
\begin{aligned}
& \left|\frac{1}{a_{n}} E\left[I(Y \geq y) K\left(\frac{\gamma_{1}^{T}(Z-z)}{a_{n}}\right)\right]-\frac{1}{a_{n}} E\left[I(Y \geq y) K\left(\frac{\gamma_{2}^{T}(Z-z)}{a_{n}}\right)\right]\right| \\
& \quad \leq c_{1}\left\|\gamma_{1}-\gamma_{2}\right\|,
\end{aligned}
$$

for universal constants $c$ and $c_{1}$. If we choose $\delta_{n} / a_{n}^{2} \rightarrow 0$ as $n \rightarrow \infty$, then for any $\delta>0$,

$$
\begin{aligned}
& P\left(\sup _{\gamma, y, z} \mid \frac{1}{n a_{n}} \sum_{j} I\left(Y_{j} \geq y\right) K\left(\frac{\gamma^{T}\left(Z_{j}-z\right)}{a_{n}}\right)\right. \\
&\left.\quad-\frac{1}{a_{n}} E\left[I(Y \geq y) K\left(\frac{\gamma^{T}(Z-z)}{a_{n}}\right)\right] \mid>\delta\right) \\
& \leq P\left(\max _{1 \leq l \leq m_{n}^{*}} \sup _{y, z} \mid \frac{1}{n a_{n}} \sum_{j} I\left(Y_{j} \geq y\right) K\left(\frac{\gamma^{(l)^{T}}\left(Z_{j}-z\right)}{a_{n}}\right)\right. \\
&\left.-\frac{1}{a_{n}} E\left[I(Y \geq y) K\left(\frac{\gamma^{(l)^{T}}(Z-z)}{a_{n}}\right)\right] \mid>\frac{\delta}{2}\right) \\
& \leq \sum_{l=1}^{m_{n}^{*}} P\left(\sup _{y, z} \mid \frac{1}{n a_{n}} \sum_{j} I\left(Y_{j} \geq y\right) K\left(\frac{\gamma^{(l)^{T}}\left(Z_{j}-z\right)}{a_{n}}\right)\right. \\
&\left.-\frac{1}{a_{n}} E\left[I(Y \geq y) K\left(\frac{\gamma^{(l)^{T}}(Z-z)}{a_{n}}\right)\right] \mid>\frac{\delta}{2}\right) \\
& \leq c_{0} m_{n}^{*} \exp \left(-c_{1} n \delta^{2} a_{n}^{2}\right),
\end{aligned}
$$

where the exponential bound in the last step makes use of the result on the empirical CDF due to Dvoretzky et al. [9]. Therefore, 


$$
\begin{aligned}
& \sum_{n=1}^{\infty} P\left(\sup _{\gamma, y, z} \mid \frac{1}{n a_{n}} \sum_{j} I\left(Y_{j} \geq y\right) K\left(\frac{\gamma^{T}\left(Z_{j}-z\right)}{a_{n}}\right)\right. \\
& \left.\quad-\frac{1}{a_{n}} E\left[I(Y \geq y) K\left(\frac{\gamma^{T}(Z-z)}{a_{n}}\right)\right] \mid>\delta\right) \\
& \leq c_{2} \sum_{n=1}^{\infty} \delta_{n}^{-q} \exp \left(-c_{1} n \delta^{2} a_{n}^{2}\right) .
\end{aligned}
$$

If we choose $\delta_{n}=a_{n}^{3}$, then the previous display becomes

$$
c_{2} \sum_{n=1}^{\infty} \frac{a_{n}^{-3 q}}{e^{c_{1} n \delta^{2} a_{n}^{2}}} \leq c_{3} \sum_{n=1}^{\infty} \frac{a_{n}^{-3 q}}{\left(n a_{n}^{2}\right)^{m}}
$$

for any positive integer $m$. Since $a_{n}=n^{\nu_{1}}$ with $v_{1} \in(-1 / 2,0)$, we can choose $m$ to be larger than $\left(1-3 q v_{1}\right) /\left(1+2 v_{1}\right)$ such that the previous display is finite. Then, by the Borel-Cantelli lemma,

$$
\sup _{\gamma, y, z}\left|\frac{1}{n a_{n}} \sum_{Y_{j} \geq y} K\left(\frac{\gamma^{T}\left(Z_{j}-z\right)}{a_{n}}\right)-\frac{1}{a_{n}} E\left[I(Y \geq y) K\left(\frac{\gamma^{T}(Z-z)}{a_{n}}\right)\right]\right| \longrightarrow_{a . s .} 0 .
$$

For any fixed $\gamma$, it can be shown that

$$
\begin{aligned}
& \frac{1}{a_{n}} E\left[I(Y \geq y) K\left(\frac{\gamma^{T}(Z-z)}{a_{n}}\right)\right] \\
& \quad=E\left(I(Y \geq y) \mid \gamma^{T} Z=\gamma^{T} z\right) f_{\gamma^{T} Z}\left(\gamma^{T} z\right)+O\left(a_{n}^{2}\right),
\end{aligned}
$$

where $f_{\gamma^{T} Z}(\cdot)$ is the density function of $\gamma^{T} Z$ and $O\left(a_{n}^{2}\right)$ does not depend on $y$ and $z$. Hence for any given $\gamma$,

$$
\begin{aligned}
\sup _{y, z}\left|\frac{1}{a_{n}} E\left[I(Y \geq y) K\left(\frac{\gamma^{T}(Z-z)}{a_{n}}\right)\right]-E\left(I(Y \geq y) \mid \gamma^{T} Z=\gamma^{T} z\right) f_{\gamma^{T} Z}\left(\gamma^{T} z\right)\right| \\
\longrightarrow 0 .
\end{aligned}
$$

Note that both $1 / a_{n} E\left[I(Y \geq y) K\left(\frac{\gamma^{T}(Z-z)}{a_{n}}\right)\right]$ and $E\left(I(Y \geq y) \mid \gamma^{T} Z=\gamma^{T} z\right) f_{\gamma^{T} Z} \times$ $\left(\gamma^{T} z\right)$ are equi-continuous in $\gamma$. Hence,

$$
\begin{aligned}
& \sup _{\gamma, y, z}\left|\frac{1}{a_{n}} E\left[I(Y \geq y) K\left(\frac{\gamma^{T}(Z-z)}{a_{n}}\right)\right]-E\left(I(Y \geq y) \mid \gamma^{T} Z=\gamma^{T} z\right) f_{\gamma^{T} Z}\left(\gamma^{T} z\right)\right| \\
& \quad \longrightarrow 0 .
\end{aligned}
$$

Therefore, we have proved that $\sup _{\gamma, y, z}\left|\frac{1}{n a_{n}} \sum_{Y_{j} \geq y} K\left(\frac{\gamma^{T}\left(Z_{j}-z\right)}{a_{n}}\right)-E\left(I(Y \geq y) \mid \gamma^{T} Z=\gamma^{T} z\right) f_{\gamma^{T} Z}\left(\gamma^{T} z\right)\right| \longrightarrow_{a . s .} 0$. 
It then follows that

$$
\begin{aligned}
& \sup _{\gamma}\left|(B)-\frac{1}{n} \sum_{j=1}^{n} \Delta_{j} \frac{1}{n a_{n}} \sum_{i=1}^{n} \frac{I\left(Y_{i} \geq Y_{j}\right) K\left(\frac{\gamma^{T}\left(Z_{i}-Z_{j}\right)}{a_{n}}\right)}{\left.E\left(I(Y \geq y) \mid \gamma^{T} Z=\gamma^{T} Z_{i}\right)\right|_{y=Y_{j}} f_{\gamma^{T}} Z\left(\gamma^{T} Z_{i}\right)}\right| \\
& \quad \longrightarrow \text { a.s. } 0 .
\end{aligned}
$$

Similar arguments can be used to show that

$$
\begin{aligned}
\sup _{\gamma, y, z} \mid \frac{1}{n a_{n}} \sum_{i=1}^{n} \frac{I\left(Y_{i} \geq y\right) K\left(\frac{\gamma^{T}\left(Z_{i}-z\right)}{a_{n}}\right)}{E\left(I(Y \geq y) \mid \gamma^{T} Z=\gamma^{T} Z_{i}\right) f_{\gamma^{T} Z}\left(\gamma^{T} Z_{i}\right)} \\
-\frac{1}{a_{n}} E\left[\frac{I(Y \geq y) K\left(\frac{\gamma^{T}(Z-z)}{a_{n}}\right)}{E\left(I(Y \geq y) \mid \gamma^{T} Z\right) f_{\gamma^{T} Z}\left(\gamma^{T} Z\right)}\right] \mid \longrightarrow_{\text {a.s. }} 0 .
\end{aligned}
$$

Simple calculation shows that the second term inside the absolute value equals 1 . Therefore,

$$
\begin{aligned}
& \sup _{\gamma}\left|\frac{1}{n} \sum_{j=1}^{n} \Delta_{j} \frac{1}{n a_{n}} \sum_{i=1}^{n} \frac{I\left(Y_{i} \geq Y_{j}\right) K\left(\frac{\gamma^{T}\left(Z_{i}-Z_{j}\right)}{a_{n}}\right)}{\left.E\left(I(Y \geq y) \mid \gamma^{T} Z=\gamma^{T} Z_{i}\right)\right|_{y=Y_{j}} f_{\gamma^{T} Z}\left(\gamma^{T} Z_{i}\right)}-\frac{1}{n} \sum_{j=1}^{n} \Delta_{j}\right| \\
& \longrightarrow \text { a.s. } 0
\end{aligned}
$$

and thus $\sup _{\gamma}\left|(B)-1 / n \sum_{j=1}^{n} \Delta_{j}\right| \longrightarrow$ a.s. 0 .

Proof of Theorem 2 Following the proof for Theorem 1, we obtain

$$
\begin{aligned}
\sup _{\gamma} \mid \frac{1}{n} \sum_{i} \Delta_{i} \log \left(\frac{1}{n a_{n}} \sum_{Y_{j} \geq Y_{i}} K\left(\frac{\gamma^{T}\left(Z_{j}-Z_{i}\right)}{a_{n}}\right)\right) \\
\quad-\frac{1}{n} \sum_{i} \Delta_{i} \log \left(\left.E\left(I(Y \geq y) \mid \gamma^{T} Z=\gamma^{T} Z_{i}\right)\right|_{y=Y_{i}} f_{\gamma^{T} Z}\left(\gamma^{T} Z_{i}\right)\right) \mid \longrightarrow_{\text {a.s. }} 0 .
\end{aligned}
$$

The second term inside the absolute value converges uniformly in $\gamma$ to

$$
E\left\{\Delta \log \left(\left.E\left(I(Y \geq y) \mid \gamma^{T} Z\right)\right|_{y=Y} f_{\gamma^{T} Z}\left(\gamma^{T} Z\right)\right)\right\},
$$

since the involved class of functions is strong P-GC. Therefore, $\sup _{\gamma} \mid p l_{n}^{\text {loc }}(\gamma)-$ $p l^{\mathrm{loc}}(\gamma) \mid \rightarrow$ a.s. 0 .

Proof of Theorem 3 Note that $-\left.\frac{\partial}{\partial \gamma}\right|_{\gamma=\gamma_{0}} p l^{\text {loc }}(\gamma)$ equals

$$
\begin{aligned}
& E\left[\left.\Delta \frac{\nabla_{\gamma}\left(E\left(I(Y \geq y) \mid \gamma^{T} Z\right) f_{\gamma^{T} Z}\left(\gamma^{T} Z\right)\right)}{E\left(I(Y \geq y) \mid \gamma_{0}^{T} Z\right) f_{\gamma_{0}^{T} Z}\left(\gamma_{0}^{T} Z\right)}\right|_{y=Y}\right] \\
& \quad=E_{Z}\left[\int \frac{\nabla_{\gamma}\left(E\left(I(Y \geq t) \mid \gamma^{T} Z\right) f_{\gamma^{T} Z}\left(\gamma^{T} Z\right)\right)}{E\left(I(Y \geq t) \mid \gamma_{0}^{T} Z\right) f_{\gamma_{0}^{T} Z}\left(\gamma_{0}^{T} Z\right)} \lambda_{0}\left(t, \gamma_{0}^{T} Z\right) G_{C}(t)\right.
\end{aligned}
$$




$$
\begin{aligned}
& \left.\times \exp \left(-\Lambda_{0}\left(t, \gamma_{0}^{T} Z\right)\right) d t\right] \\
= & E_{Z}\left[\int \frac{\nabla_{\gamma}\left(E\left(I(Y \geq t) \mid \gamma^{T} Z\right) f_{\gamma^{T} Z}\left(\gamma^{T} Z\right)\right)}{f_{\gamma_{0}^{T} Z}\left(\gamma_{0}^{T} Z\right)} \lambda_{0}\left(t, \gamma_{0}^{T} Z\right) d t\right] \\
= & \iint \frac{\lambda_{0}\left(y, \gamma_{0}^{T} w\right) f_{Z}(w)}{f_{\gamma_{0}^{T} Z}\left(\gamma_{0}^{T} w\right)} \nabla_{\gamma}\left(E\left(I(Y \geq y) \mid \gamma^{T} Z=\gamma^{T} w\right) f_{\gamma^{T} Z}\left(\gamma^{T} w\right)\right) d y d w,
\end{aligned}
$$

where $G_{C}(\cdot)$ denotes the survival function of $C$. The quantity inside the gradient operator can be written as

$$
\begin{aligned}
& \lim _{h \rightarrow 0} \frac{1}{h} E\left[I(Y \geq y) K\left(\frac{\gamma^{T} Z-\gamma^{T} w}{h}\right)\right] \\
& \quad=\lim _{h \rightarrow 0} \frac{1}{h} E_{Z}\left[K\left(\frac{\gamma^{T} Z-\gamma^{T} w}{h}\right) g\left(y, \gamma_{0}^{T} Z\right)\right],
\end{aligned}
$$

where $g(y, u)=G_{C}(y) \exp \left(-\Lambda_{0}(y, u)\right)$. Thus

$$
\begin{aligned}
-\left.\frac{\partial}{\partial \gamma}\right|_{\gamma_{0}} p l^{\mathrm{loc}}(\gamma)= & \iint \frac{\lambda_{0}\left(y, \gamma_{0}^{T} w\right) f_{Z}(w)}{f_{\gamma_{0}^{T} Z}\left(\gamma_{0}^{T} w\right)} \\
& \times \lim _{h \rightarrow 0} E_{Z}\left[\frac{1}{h^{2}} K^{\prime}\left(\frac{\gamma_{0}^{T} Z-\gamma_{0}^{T} w}{h}\right)(Z-w) g\left(y, \gamma_{0}^{T} Z\right)\right] d y d w \\
= & \iint \frac{\lambda_{0}\left(y, \gamma_{0}^{T} w\right) f_{Z}(w)}{f_{\gamma_{0}^{T} Z}\left(\gamma_{0}^{T} w\right)} \lim _{h \rightarrow 0} E_{\gamma_{0}^{T} Z}\left[\frac{1}{h^{2}} K^{\prime}\left(\frac{\gamma_{0}^{T} Z-\gamma_{0}^{T} w}{h}\right)\right. \\
& \left.\times\left(E\left(Z \mid \gamma_{0}^{T} Z\right)-w\right) g\left(y, \gamma_{0}^{T} Z\right)\right] d y d w .
\end{aligned}
$$

Let $r(u) \equiv E\left(Z \mid \gamma_{0}^{T} Z=u\right)$, then the limit inside of the integral is

$$
\begin{aligned}
\lim _{h \rightarrow 0} \int & \frac{1}{h^{2}} K^{\prime}\left(\frac{u-\gamma_{0}^{T} w}{h}\right)(r(u)-w) g(y, u) f_{\gamma_{0}^{T} Z}(u) d u \\
= & -g_{2}^{\prime}\left(y, \gamma_{0}^{T} w\right) f_{\gamma_{0}^{T} Z}\left(\gamma_{0}^{T} w\right) r\left(\gamma_{0}^{T} w\right)-g\left(y, \gamma_{0}^{T} w\right) f_{\gamma_{0}^{T} Z}^{\prime}\left(\gamma_{0}^{T} w\right) r\left(\gamma_{0}^{T} w\right) \\
& -g\left(y, \gamma_{0}^{T} w\right) f_{\gamma_{0}^{T} Z}\left(\gamma_{0}^{T} w\right) r^{\prime}\left(\gamma_{0}^{T} w\right)+g_{2}^{\prime}\left(y, \gamma_{0}^{T} w\right) f_{\gamma_{0}^{T} Z}\left(\gamma_{0}^{T} w\right) w \\
& +g\left(y, \gamma_{0}^{T} w\right) f_{\gamma_{0}^{T} Z}^{\prime}\left(\gamma_{0}^{T} w\right) w,
\end{aligned}
$$


where $g_{2}^{\prime}(y, u)=\frac{\partial}{\partial u} g(y, u)$. Hence, the double integral equals

$$
\begin{aligned}
\int E_{Z}\left[\lambda _ { 0 } ( y , \gamma _ { 0 } ^ { T } Z ) \left(-g_{2}^{\prime}\left(y, \gamma_{0}^{T} Z\right) r\left(\gamma_{0}^{T} Z\right)-g\left(y, \gamma_{0}^{T} Z\right) \frac{f_{\gamma_{0}^{T} Z}^{\prime}}{f_{\gamma_{0}^{T} Z}}\left(\gamma_{0}^{T} Z\right) r\left(\gamma_{0}^{T} Z\right)\right.\right. \\
\left.\left.\quad-g\left(y, \gamma_{0}^{T} Z\right) r^{\prime}\left(\gamma_{0}^{T} Z\right)+g_{2}^{\prime}\left(y, \gamma_{0}^{T} Z\right) Z+g\left(y, \gamma_{0}^{T} Z\right) \frac{f_{\gamma_{0}^{T} Z}^{\prime}}{f_{\gamma_{0}^{T} Z}}\left(\gamma_{0}^{T} Z\right) Z\right)\right] d y \\
=-\int E_{\gamma_{0}^{T} Z}\left(\lambda_{0}\left(y, \gamma_{0}^{T} Z\right) g\left(y, \gamma_{0}^{T} Z\right) r^{\prime}\left(\gamma_{0}^{T} Z\right)\right) d y .
\end{aligned}
$$

Since $Z \sim N(\mu, \Sigma), r^{\prime}(u)=\left(\gamma_{0}^{T} \Sigma \gamma_{0}\right)^{-1} \Sigma \gamma_{0}$ for any u. Thus the last display becomes

$$
-\left(\gamma_{0}^{T} \Sigma \gamma_{0}\right)^{-1} \Sigma \gamma_{0} \int E_{\gamma_{0}^{T} Z}\left(\lambda_{0}\left(y, \gamma_{0}^{T} Z\right) g\left(y, \gamma_{0}^{T} Z\right)\right) d y=-E[\Delta]\left(\gamma_{0}^{T} \Sigma \gamma_{0}\right)^{-1} \Sigma \gamma_{0}
$$

By (C6), $E[\Delta]>0$. Thus the display is proportional to $\gamma_{0}$ if and only if $\Sigma \gamma_{0} \propto c \gamma_{0}$ for some constant $\mathrm{c}$. This completes the proof.

Proof of Theorem $4-p l_{n}^{s}(\gamma)$ is equal to

$$
\sum_{k=1}^{J_{n}} \mathbb{P}_{n}\left[\Delta I\left(\mathbb{F}_{n}\left(\gamma^{T} Z\right) \in S_{k}\right) \log \left(\frac{J_{n}}{n} \sum_{j} I\left(Y_{j} \geq Y\right) I\left(\mathbb{F}_{n}\left(\gamma^{T} Z_{j}\right) \in S_{k}\right)\right)\right]
$$

Since $J_{n} / \sqrt{n} \rightarrow 0$ and by Donsker arguments, we have

$$
\left|\frac{J_{n}}{n} \sum_{j} I\left(Y_{j} \geq y\right) I\left(\mathbb{F}_{n}\left(\gamma^{T} Z_{j}\right) \in S_{k}\right)-J_{n} E\left[I(Y \geq y) I\left(F_{\gamma^{T} Z}\left(\gamma^{T} Z\right) \in S_{k}\right)\right]\right| \stackrel{P}{\longrightarrow} 0,
$$

uniformly in $y$ and $\gamma$, where $F_{\gamma^{T} Z}(\cdot)$ is the distribution function of $\gamma^{T} Z$. Note that

$$
\begin{array}{rl}
J_{n} & E\left(I(Y \geq y) I\left(F_{\gamma^{T} Z}\left(\gamma^{T} Z\right) \in S_{k}\right)\right) \\
& =E\left[I(Y \geq y) \mid F_{\gamma^{T} Z}\left(\gamma^{T} Z\right)=(k-1) / J_{n}\right]+o(1) .
\end{array}
$$

Hence,

$$
\begin{aligned}
& \left|\frac{J_{n}}{n} \sum_{j} I\left(Y_{j} \geq y\right) I\left(\mathbb{F}_{n}\left(\gamma^{T} Z_{j}\right) \in S_{k}\right)-E\left[I(Y \geq y) \mid F_{\gamma^{T} Z}\left(\gamma^{T} Z\right)=(k-1) / J_{n}\right]\right| \\
& \quad \stackrel{P}{\longrightarrow} 0
\end{aligned}
$$


uniformly in $y$ and $\gamma$. Next, by either Glivenko-Cantelli or Donsker arguments,

$$
\begin{aligned}
\mid- & p l_{n}^{S}(\gamma)-\sum_{k=1}^{J_{n}} E\left[\Delta I ( F _ { \gamma ^ { T } Z } ( \gamma ^ { T } Z ) \in S _ { k } ) \operatorname { l o g } E \left[I(Y \geq y) \mid F_{\gamma^{T} Z}\left(\gamma^{T} Z\right)\right.\right. \\
& \left.\left.=(k-1) / J_{n}\right]\left.\right|_{y=Y}\right] \mid \stackrel{P}{\longrightarrow} 0,
\end{aligned}
$$

uniformly in $\gamma$. The second term inside the absolute value of the above display equals

$$
\sum_{k=1}^{J_{n}} \frac{1}{J_{n}} E\left[\left.\Delta \log E\left[I(Y \geq y) \mid F_{\gamma^{T} Z}\left(\gamma^{T} Z\right)\right]\right|_{y=Y} \mid F_{\gamma^{T} Z}\left(\gamma^{T} Z\right)=(k-1) / J_{n}\right]
$$

which converges to

$$
\begin{aligned}
& \int_{0}^{1} E\left[\left.\Delta \log E\left[I(Y \geq y) \mid F_{\gamma^{T} Z}\left(\gamma^{T} Z\right)\right]\right|_{y=Y} \mid F_{\gamma^{T} Z}\left(\gamma^{T} Z\right)=u\right] f_{F_{\gamma^{T} Z}\left(\gamma^{T} Z\right)}(u) d u \\
& =E\left\{E\left[\left.\Delta \log E\left[I(Y \geq y) \mid F_{\gamma^{T} Z}\left(\gamma^{T} Z\right)\right]\right|_{y=Y} \mid F_{\gamma^{T} Z}\left(\gamma^{T} Z\right)\right]\right\} \\
& =E\left[\left.\Delta \log E\left[I(Y \geq y) \mid F_{\gamma^{T} Z}\left(\gamma^{T} Z\right)\right]\right|_{y=Y}\right] \text {. }
\end{aligned}
$$

Hence, we have shown that $p l_{n}^{s}(\gamma)$ converges uniformly in $\gamma$ to $p l^{s}(\gamma)$.

Proof of Theorem 5 By Theorem 2.12 of Kosorok [13] and Theorem 4, it suffices to show that $\gamma_{0}$ is the unique maximizer of $p l^{s}(\gamma)$. Since

$$
\begin{aligned}
p l^{s}(\gamma)= & -E\left[\int \log P\left(Y \geq t \mid \gamma^{T} Z\right) f_{T \mid Z}(t) G_{C}(t) d t\right] \\
= & -E\left[\int \log \left(G_{C}(t) E\left(S_{T \mid Z}(t) \mid \gamma^{T} Z\right)\right) f_{T \mid Z}(t) G_{C}(t) d t\right] \\
= & -E\left[\int \log E\left(S_{T \mid Z}(t) \mid \gamma^{T} Z\right) f_{T \mid Z}(t) G_{C}(t) d t\right] \\
& -E\left[\int \log G_{C}(t) f_{T \mid Z}(t) G_{C}(t) d t\right] \\
= & -E\left[\int\left(E\left(f_{T \mid Z}(t) \mid \gamma^{T} Z\right) \log E\left(S_{T \mid Z}(t) \mid \gamma^{T} Z\right)\right) G_{C}(t) d t\right] \\
& -E\left[\int \log G_{C}(t) f_{T \mid Z}(t) G_{C}(t) d t\right]
\end{aligned}
$$

and 


$$
\begin{aligned}
& E\left(f_{T \mid Z}(t) \mid \gamma^{T} Z\right) \log E\left(S_{T \mid Z}(t) \mid \gamma^{T} Z\right) \\
&=-\frac{d}{d t}\left[E\left(S_{T \mid Z}(t) \mid \gamma^{T} Z\right) \log E\left(S_{T \mid Z}(t) \mid \gamma^{T} Z\right)\right]-E\left(f_{T \mid Z}(t) \mid \gamma^{T} Z\right) \\
& p l^{S}(\gamma)=\int \frac{d}{d t}\left\{E\left[E\left(S_{T \mid Z}(t) \mid \gamma^{T} Z\right) \log E\left(S_{T \mid Z}(t) \mid \gamma^{T} Z\right)\right]\right\} G_{C}(t) d t \\
&+\int E\left[f_{T \mid Z}(t)\right] G_{C}(t) d t-E\left[\int \log G_{C}(t) f_{T \mid Z}(t) G_{C}(t) d t\right]
\end{aligned}
$$

The first term of the previous display equals

$$
\begin{aligned}
\int G_{C}(t) d E\left[E\left(S_{T \mid Z}(t) \mid \gamma^{T} Z\right) \log E\left(S_{T \mid Z}(t) \mid \gamma^{T} Z\right)\right] \\
=\int E\left[E\left(S_{T \mid Z}(t) \mid \gamma^{T} Z\right) \log E\left(S_{T \mid Z}(t) \mid \gamma^{T} Z\right)\right] f_{C}(t) d t \\
\leq \int E\left[E\left(S_{T \mid Z}(t) \log S_{T \mid Z}(t) \mid \gamma^{T} Z\right)\right] f_{C}(t) d t \\
=\int E\left(S_{T \mid Z}(t) \log S_{T \mid Z}(t)\right) f_{C}(t) d t
\end{aligned}
$$

where the inequality follows from Jensen's inequality since $g(x) \equiv x \log x$ is a convex function. Therefore, for any $\gamma, p l^{S}(\gamma)$ is less than or equal to

$$
\begin{aligned}
\int & E\left(S_{T \mid Z}(t) \log S_{T \mid Z}(t)\right) f_{C}(t) d t \\
& +\int E\left[f_{T \mid Z}(t)\right] G_{C}(t) d t-E\left[\int \log G_{C}(t) f_{T \mid Z}(t) G_{C}(t) d t\right] \\
= & -\int E\left(S_{T \mid Z}(t) \log S_{T \mid Z}(t)\right) d G_{C}(t) \\
& +\int E\left[f_{T \mid Z}(t)\right] G_{C}(t) d t-E\left[\int \log G_{C}(t) f_{T \mid Z}(t) G_{C}(t) d t\right] \\
= & \int G_{C}(t) d E\left(S_{T \mid Z}(t) \log S_{T \mid Z}(t)\right) \\
& +\int E\left[f_{T \mid Z}(t)\right] G_{C}(t) d t-E\left[\int \log G_{C}(t) f_{T \mid Z}(t) G_{C}(t) d t\right] \\
= & -\int G_{C}(t) E\left[f_{T \mid Z}(t) \log P(Y \geq t \mid Z)\right] d t \\
= & -E\left[\left.\Delta \log P\left(Y \geq t \mid \gamma_{0}^{T} Z\right)\right|_{t=Y}\right]=p l^{s}\left(\gamma_{0}\right) .
\end{aligned}
$$

Suppose $p l^{S}\left(\gamma^{*}\right)=p l^{S}\left(\gamma_{0}\right)$, then conditional on $\gamma^{* T} Z, S_{T \mid Z}(t)$ is a constant almost surely since the function $g(\cdot)$ is strictly convex. That is, $S_{T \mid Z}(t)=h\left(t, \gamma^{* T} Z\right)$ almost 
surely for some function $h(\cdot)$. After taking the derivative with respect to $Z$ on both sides, we obtain $\gamma^{*} \propto \gamma_{0}$. The proof is complete in view of the requirements that $\gamma^{*}$ has a unit norm with one positive component.

\section{References}

1. Andersen, P.K., Borgan, Ø., Gill, R.D., Keiding, N.: Statistical Models Based on Counting Processes. Springer, New York (1993)

2. Andersen, P.K., Gill, R.D.: Cox's regression model for counting processes: a large sample study. Ann. Stat. 10, 1100-1120 (1982)

3. Bennett, S.: Analysis of survival data by the proportional odds model. Stat. Med. 2, 273-277 (1983)

4. Carroll, R.J., Fan, J., Gijbels, I., Wand, M.P.: Generalized partially linear single-index models. J. Am. Stat. Assoc. 92, 477-489 (1997)

5. Chow, T., Kereiakes, D.J., Bartone, C., Booth, T., Schloss, E.J., Waller, T., Chung, E.S., Menon, S., Nallamothu, B.K., Chan, P.S.: Prognostic utility of microvolt t-wave alternans in risk stratification of patients with ischemic cardiomyopathy. J. Am. Coll. Cardiol. 47, 1820-1827 (2006)

6. Cox, D.R.: Regression models and life tables (with discussion). J. R. Stat. Soc. B 34, 187-220 (1972)

7. Cox, D.R.: Partial likelihood. Biometrika 62, 269-276 (1975)

8. Cox, D.R., Oakes, D.: Analysis of Survival Data. Chapman \& Hall, London (1984)

9. Dvoretzky, A., Kiefer, J., Wolfowitz, J.: Asymptotic minimax character of the sample distribution function and of the classical multinomial estimator. Ann. Math. Stat. 27, 642-669 (1956)

10. Fan, J., Gijbels, I., King, M.: Local likelihood and local partial likelihood in hazard regression. Ann. Stat. 25, 1661-1690 (1997)

11. Fleming, T.R., Harrington, D.P.: Counting Processes and Survival Analysis. Wiley, New York (1991)

12. Härdle, W., Hall, P., Ichimura, H.: Optimal smoothing in single-index models. Ann. Stat. 21, 157-178 (1993)

13. Kosorok, M.R.: Introduction to Empirical Processes and Semiparametric Inference. Springer, New York (2008)

14. Kosorok, M.R., Lee, B.L., Fine, J.P.: Robust inference for univariate proportional hazards frailty regression models. Ann. Stat. 32, 1448-1491 (2004)

15. Motzer, R.J., Mazumdar, M., Bacik, J., Berg, W., Amsterdam, A., Ferrara, J.: Survival and prognostic stratification of 670 patients with advanced renal cell carcinoma. J. Clin. Oncol. 17, 2530-2540 (1999)

16. Nielsen, J.P., Linton, O.B.: Kernel estimation in a nonparametric marker dependent hazard model. Ann. Stat. 23, 1735-1748 (1995)

17. Pettitt, A.N.: Proportional odds models for survival data and estimates using ranks. Appl. Stat. 33, 169-175 (1984)

18. Yu, Y., Ruppert, D.: Penalized spline estimation for partially linear single-index models. J. Am. Stat. Assoc. 97, 1042-1054 (2002)

19. Zeng, D., Lin, D.Y.: Maximum likelihood estimation in semiparametric regression models with censored data (with discussion). J. R. Stat. Soc. B 69, 507-564 (2007) 